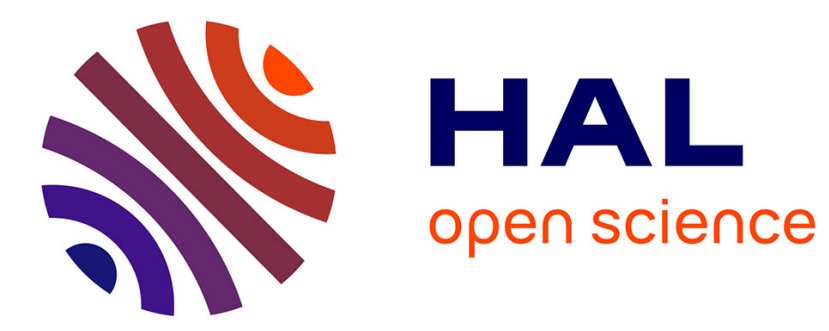

\title{
Correlation dimension of self-similar surfaces and application to Kirchhoff integrals
}

Charles-Antoine Guérin, Matthias Holschneider

\section{To cite this version:}

Charles-Antoine Guérin, Matthias Holschneider. Correlation dimension of self-similar surfaces and application to Kirchhoff integrals. Journal of Physics A: Mathematical and General (1975 - 2006), 2003, 36 (34), pp.9067-9079. 10.1088/0305-4470/36/34/309 . hal-00083207

\section{HAL Id: hal-00083207 https://hal.science/hal-00083207}

Submitted on $10 \mathrm{Jul} 2016$

HAL is a multi-disciplinary open access archive for the deposit and dissemination of scientific research documents, whether they are published or not. The documents may come from teaching and research institutions in France or abroad, or from public or private research centers.
L'archive ouverte pluridisciplinaire HAL, est destinée au dépôt et à la diffusion de documents scientifiques de niveau recherche, publiés ou non, émanant des établissements d'enseignement et de recherche français ou étrangers, des laboratoires publics ou privés. 


\title{
Correlation dimension of self-similar surfaces and application to Kirchhoff integrals
}

\author{
Charles-Antoine Guérin ${ }^{1}$ and Matthias Holschneider ${ }^{2,3}$ \\ ${ }^{1}$ Institut Fresnel, UMR CNRS 6133, Faculté des Sciences de Saint-Jérôme, case 162, F-13397 Marseille cedex 20, France \\ 2 Universtität Potsdam, Applied and Industrial Mathematics, PF 6015 53, D-14415 Potsdam, Germany
}

E-mail: charles-antoine.guerin@fresnel.fr and hols@math.uni-potsdam.de

\begin{abstract}
For s urfaces generated by a class of asymptotically self-similar processes we define a probability measure, supported by the surface. We show that the correlation dimension of that surface measure is linked to the self-similarity exponent almost surely. This result is applied to the Kirchhoff integral well known in scattering from rough surfaces. We show that a certain average of the scattered intensity exhibits almost surely a scaling that allows us to recover the self-similarity index of the surface in an experiment involving only one sample of the surface.
\end{abstract}

\section{Introduction}

Random fractal models are often used to describe natural rough surfaces that exhibit structures over a wide range of scales, such as sea surfaces, soils, mountains or rough deposits. A powerful tool for the remote characterization of such media is wave scattering. This method is now commonly used in various domains such as oceanography, geophysics or physics and chemistry of solid surfaces. We refer to [1] for a general introduction to fractal geometry and to [2] and [3] for some surveys on scattering from fractal surfaces. The wavenumber of the interrogating wave is chosen in accordance with the spatial frequencies to be probed in the material. The typical observation is a power-law dependence of the scattered intensity upon the wavenumber, with a non-trivial exponent which is interpreted as a fractal dimension of the surface. Such results can be well explained in the framework of the small-perturbation model, i.e in a regime where the roughness is small with respect to the illuminating wavelength.

3 On leave from CNRS. 


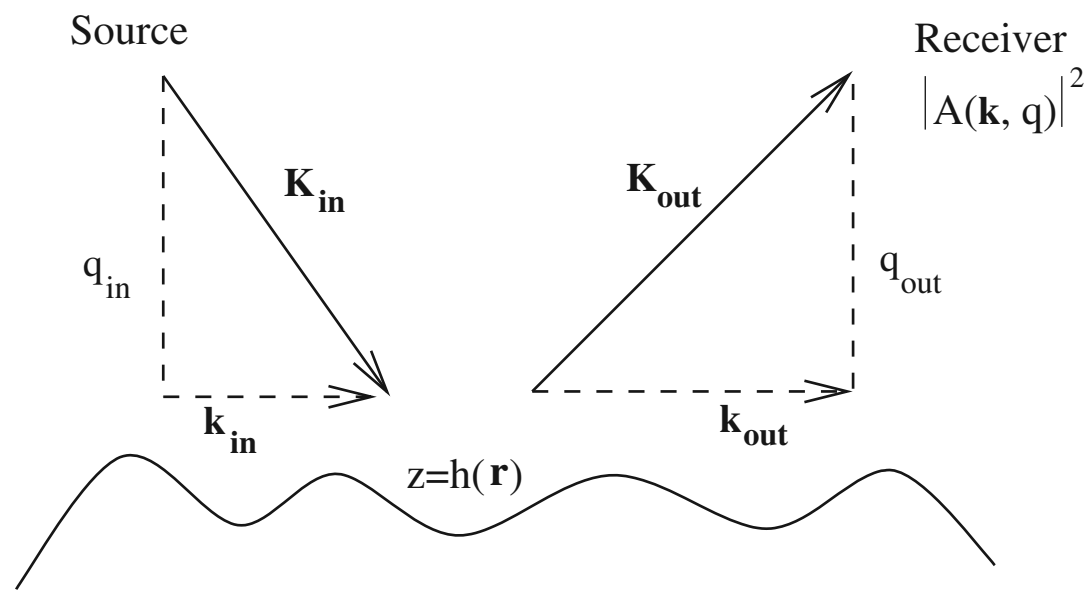

Figure 1. Scattering geometry.

In that case, the scattered amplitude is a mere Fourier transform of the surface and the mean scattered intensity yields directly its power spectrum. However, the interpretation of the scattering data becomes problematic outside the perturbative regime. Very often, the scattering amplitude is then approximated by an oscillatory integral of the following form:

$$
A(\boldsymbol{k}, q)=\int_{\mathbb{R}^{n}} \chi(\boldsymbol{r}) \mathrm{e}^{\mathrm{i}(\boldsymbol{k} \cdot \boldsymbol{r}+q h(\boldsymbol{r}))} \mathrm{d} \boldsymbol{r} .
$$

Here $h(\boldsymbol{r}), \boldsymbol{r} \in \mathbb{R}^{n}$ is a continuous function representing the rough interface, $\chi$ is a cut-off function that delimits the illuminated area, $A$ is the scattered amplitude for a fixed sourcereceiver geometry and $(\boldsymbol{k}, q) \in \mathbb{R}^{n} \times \mathbb{R}$ are the horizontal and vertical frequency variables, respectively. They are related to the components of the incoming and outcoming wave vectors by $\boldsymbol{k}=\boldsymbol{k}_{\text {out }}-\boldsymbol{k}_{\text {in }}$ and $q=q_{\text {in }}+q_{\text {out }}$ (see figure 1). For simplicity, $\chi$ will be chosen as the (normalized) characteristic function of the unit sphere. We call an integral of type (1.1) a 'Kirchhoff integral'. This terminology originates from the so-called Kirchhoff approximation [4], which leads to this expression for the scattering amplitude, apart from trivial geometrical factors. The Kirchhoff approximation amounts to replacing the surface by its local tangent plane in the calculation of the unknown field at the boundary. This simplifying assumption is justified for smooth surfaces but is in principle incompatible with fractal surfaces, where such a tangent plane does not even exist. Many authors have chosen to work with band-limited fractal surfaces, but still the use of the Kirchhoff approximation is questionable since the frequency domain where the method applies and the fractal frequency regime do not necessarily overlap. A second instance where this integral appears is the case of two homogeneous media separated by a rough interface $h$ and illuminated from one side, say the upper medium. In the limit of small contrast the scattering amplitude is accurately given by the so-called Born approximation, which amounts to replacing the total field in the lower medium by the incident field. The Born approximation in this context takes on the form of a Kirchhoff integral. Scattering from fractal surfaces under the Kirchhoff approximation has been extensively studied in the physical literature (e.g., [5-12]). Typically, the diffraction diagram retains the fractal properties of the surface and a dependence of the scattered intensity upon some fractal dimension is evidenced numerically or analytically in different regimes.

The aim of this work is to establish a precise and quantitative link between the fractal properties of $h$ and the asymptotic behaviour of the Kirchhoff type oscillatory integral $A$ as $(\boldsymbol{k}, q) \rightarrow \infty$. As we want to show now, the theoretical results of this paper allow us to recover the self-similarity exponent of an asymptotically self-similar random surface with probability 
one by a scattering experiment, where only the intensity is measured. This is important, since it shows that only one sample of the surface is needed (instead of a characteristic ensemble). The physical problem corresponds to $n=1$ or $n=2$, i.e. when $h$ actually describes a profile or a surface.

However, the results of this paper hold and will be presented in arbitrary dimension $n \geqslant 1$. Note that $A$ is the $(n+1)$-dimensional Fourier transform of the measure

$$
\mu=\chi(\boldsymbol{r}) \delta(z-h(\boldsymbol{r}))
$$

which is supported by the graph of $h$. Thus, at the mathematical level we are interested in how the high-frequency behaviour of the Fourier transform of $\mu$ is related to small-scale properties of $h$. The link will be established through the correlation dimension of the graph measure. The main results will be exposed in detail in the following section.

\section{Exposition of the main results}

\subsection{The graph measure}

Consider the graph $\Gamma \subset \mathbb{R}^{n+1}$ of a continuous real valued function $z=h(r)$ defined on the unit ball $\|\boldsymbol{r}\| \leqslant 1$ in $\mathbb{R}^{n}, \Gamma=\{(\boldsymbol{r}, z) \mid z=h(\boldsymbol{r}),\|\boldsymbol{r}\| \leqslant 1\}$. A natural question is to understand how fractal properties of $h$ are mirrored in fractal properties of $\Gamma$. When $h$ is fractional Brownian motion (fBm) over $\mathbb{R}$ it is known that almost surely the Hausdorff-Besicovitch dimension $D_{\mathrm{HB}}$ of the graph is simply related to the Hurst exponent $H$ by $D_{\mathrm{HB}}=2-H$ [13].

In this paper we will prove a similar result for the correlation dimension of the measure supported by $\Gamma$ which is defined as follows. Consider the extension to all of $\mathbb{R}^{n+1}$ of the pullback of the Lebesgue measure to $\Gamma$ under the projection

$$
\pi: \Gamma \rightarrow \mathbb{R}^{n} \quad(\boldsymbol{r}, z) \mapsto \boldsymbol{r} .
$$

More precisely, for any Borel set $B \in \mathbb{R}^{n+1}$ we set (with a normalization factor)

$$
\mu(B)=\frac{1}{V_{n}}|\pi(B \cap \Gamma)|
$$

where $|\cdot|$ denotes Lebesgue measure and $V_{n}$ is the measure of the unit ball in $\mathbb{R}^{n}$. Since $h$ is continuous, this defines a measure whose support is precisely $\Gamma$. Because of the normalization it is a probability measure. Formally we can write

$$
\mu=\chi(\boldsymbol{r}) \delta(z-h(\boldsymbol{r}))
$$

where $V_{n} \chi$ is the characteristic function of the unit ball in $\mathbb{R}^{n}$. Note that this makes sense, if we agree to integrate over $z$ first. We can also view this measure as the push forward of Lebesgue measure on $\mathbb{R}^{n}$ by the mapping $\boldsymbol{r} \rightarrow(\boldsymbol{r}, h(\boldsymbol{r}))$. Thus to draw a random point according to $\mu$, we pick a random point $\boldsymbol{r}$ uniformly in the unit ball in $\mathbb{R}^{n}$ and go to the graph above $\boldsymbol{r}$.

The main theorem of the paper can be formulated as follows. For the precise definitions of the employed terms see below.

Theorem 2.1. Let $h$ be a version of (isotropic) fractional Brownian motion over $\mathbb{R}^{n}$ with continuous trajectories. Let $\alpha$ be its self-similarity exponent. Let $\mu$ be the graph measure associated with each trajectory. Then the upper and lower correlation dimensions of $\mu$ are random variables which are constant almost surely with:

$$
d_{c}^{ \pm}[\mu]=n+1-\alpha / 2 \text {. }
$$

Actually we will show this theorem for a larger class of processes, which are only asymptotically self-similar. 


\subsection{The correlation dimension}

Here we want to recall the basic facts of correlation dimension. The correlation dimension $d_{c}(\mu)$ of a probability measure $\mu$ in $\mathbb{R}^{m}$ is defined in the following way [14]. Consider the so-called correlation integral at scale $\epsilon$ :

$$
X(\epsilon)=\int \mathrm{d} \mu(\boldsymbol{R}) \mu(B(\boldsymbol{R}, \epsilon))
$$

where $B(\boldsymbol{R}, \epsilon)$ is the ball of radius $\epsilon$ around the point $\boldsymbol{R}$. If a power-law behaviour is observed at small scales,

$$
X(\epsilon) \sim \epsilon^{d_{c}} \quad \epsilon \rightarrow 0
$$

then the corresponding exponent $d_{c}$ is termed the correlation dimension of the measure $\mu$. More precisely, the upper and lower correlation dimensions are defined as

$$
d_{c}^{ \pm}[\mu]=\lim _{\epsilon \rightarrow 0} \sup _{\inf } \frac{\log X(\epsilon)}{\log \epsilon} \quad \epsilon \rightarrow 0 .
$$

Note that $X(\epsilon)$ is the probability that two points randomly chosen in $\mathbb{R}^{m}$ according to the probability measure $\mu$ are within a mutual distance of $\epsilon$, thereby justifying the denomination. This becomes obvious on rewriting:

$$
X(\epsilon)=\int_{\mathbb{R}^{m} \times \mathbb{R}^{m}} \mathrm{~d} \mu(\boldsymbol{u}) \mathrm{d} \mu(\boldsymbol{v}) H(\epsilon-\|\boldsymbol{u}-\boldsymbol{v}\|)
$$

where $H$ is the Heaviside function. The correlation dimension is related to the HausdorffBesicovitch dimension $D_{\mathrm{HB}}(\Gamma)$ of the support of $\mu$ by the general inequality:

$$
\mathrm{d}_{c}^{+}[\mu] \leqslant D_{\mathrm{HB}}(\Gamma) \text {. }
$$

A useful property of the correlation dimension is its relation to the Fourier asymptotic of the measure. Define the Fourier transform of $\mu$ by

$$
\widehat{\mu}(\boldsymbol{\xi})=\int_{\mathbb{R}^{m}} \mathrm{~d} \mu(\boldsymbol{R}) \mathrm{e}^{-\mathrm{i} \boldsymbol{\xi} \cdot \boldsymbol{R}}
$$

and consider the Cesaro average:

$$
C(K)=\frac{1}{K^{m}} \int_{\|\boldsymbol{\xi}\| \leqslant K^{2}} \mathrm{~d} \boldsymbol{\xi}|\widehat{\mu}(\boldsymbol{\xi})|^{2} .
$$

Then it was shown in [15] that the scaling of $C(K)$ is governed by the correlation dimension of $\mu$ in the sense that

$$
\lim _{K \rightarrow \infty} \sup _{\inf } \frac{\log C(K)}{\log 1 / K}=d_{c}^{ \pm} .
$$

In view of these results, theorem 2.1 can be given the following equivalent form. For a vector $(\boldsymbol{k}, q)$ in $\mathbb{R}^{n+1}$ and the graph measure $\mu$ we note that

$$
\widehat{\mu}(\boldsymbol{k}, q)=A(\boldsymbol{k}, q) \text {. }
$$

Thus we have the immediate corollary:

Corollary 2.2. Under the conditions of theorem 2.1 we have

$$
\lim _{K \rightarrow \infty} \sup _{\inf } \frac{\log \int_{\|\boldsymbol{k}\|^{2}+q^{2} \leqslant K} \mathrm{~d} \boldsymbol{k} \mathrm{d} q|A(\boldsymbol{k}, q)|^{2}}{\log K}=\alpha / 2
$$

for almost all realizations of $h$. 


\subsection{The projected measure}

Take a probability measure $\mu$ in $\mathbb{R}^{m}$. For a fixed direction $\boldsymbol{v} \in \mathbb{R}^{m}$ with $\|\boldsymbol{v}\|=1$ consider the projection along $\boldsymbol{v}^{\perp}$ defined as follows:

$$
L: \mathbb{R}^{m} \rightarrow \mathbb{R} \quad \boldsymbol{R} \mapsto \boldsymbol{v} \cdot \boldsymbol{R} .
$$

The push forward of $\mu$ under $L$ defines a positive measure on $\mathbb{R}$, which we denote by $\mu_{v}$. More precisely, for any Borel set $B \subset \mathbb{R}$ denote by $L^{-1} B$ its pre-image under $L$. We then have

$$
\mu_{v}(B)=\mu\left(L^{-1} B\right) .
$$

Let $\Pi_{a} \subset \mathbb{R}^{m}, a \in \mathbb{R}$ be the affine hyperplane defined by $(\boldsymbol{v}, \boldsymbol{R})=a$. Then we can formally write

$$
\mu_{v}(a)=\int_{\Pi_{a}} \mu
$$

If now $\mu$ is the graph measure considered previously it seems clear that this measure again should contain information about the fractality of $h$. However, as it turns out, it is hidden in a more subtle way, since it has a trivial correlation dimension. We did not succeed in proving a corresponding version of theorem 2.1 for the measure $\mu_{v}$. However, we will prove the following weaker result:

Theorem 2.3. Under the hypothesis of theorem 2.1, let $\mu_{v}$ be the projected graph measure along direction $\boldsymbol{v}^{\perp}$. Then the upper and lower correlation dimensions of $\mu_{\boldsymbol{v}}$ are trivial in the sense that

$$
\mathbb{E} d_{c}^{ \pm}\left[\mu_{v}\right]=1
$$

\subsection{The wavelet correlation dimension}

In the case that a probability measure $v$ in $\mathbb{R}^{m}$ has a square integrable density $f$ the correlation integral always exhibits a trivial scaling in the sense that $d_{c}[v]=m$, as follows from

$$
\epsilon^{-m} X(\epsilon) \rightarrow V_{n} \int_{\mathbb{R}^{m}}|f|^{2}=: V_{m}\|\nu\|_{2}^{2} \quad \epsilon \rightarrow 0 .
$$

However, some sub-scaling may still reveal fractal properties of such a measure. The waveletcorrelation dimensions $d_{w}^{ \pm}$are a natural generalization of the standard correlation dimension $[16,17]$. For this paper it is enough to give the following definition:

$$
d_{w}[v]^{ \pm}=\lim _{\epsilon \rightarrow 0} \sup _{\inf } \frac{\log \left(X(\epsilon)-V_{m}\|v\|_{2}^{2} \epsilon^{m}\right)}{\log \epsilon} .
$$

This fractal exponent can again be seen in the Fourier domain

$$
\lim _{K \rightarrow \infty} \sup _{\inf } \frac{\log \|v\|_{2}^{2}-\log C(K)}{\log 1 / K}=\lim _{K \rightarrow \infty} \sup _{\inf } \frac{\log \int_{\|\xi\|>K}|\hat{v}(\xi)|^{2}}{\log 1 / K}=d_{w}^{ \pm}[v] .
$$

For the projected measure $\mu_{v}$ we will show the following result.

Theorem 2.4. Under the conditions of theorem 2.3 let $\mu_{v}$ be the projected graph measure along a direction $\boldsymbol{v}^{\perp}$. Then we have

$$
\lim _{a \rightarrow \infty} \sup _{\inf } \frac{\mathbb{E}\left|\widehat{\mu_{v}}(a)\right|^{2}}{\log (1 / a)}=2 n / \alpha
$$


As a consequence, the projected measure has a non-trivial wavelet correlation dimension in the sense that:

$$
\mathbb{E} d_{w}^{ \pm}\left[\mu_{v}\right] \geqslant n(2 / \alpha-1) .
$$

This will be applied to the scattering problem in the following way. For any direction $\boldsymbol{v} \in \mathbb{R}^{n+1},\|\boldsymbol{v}\|=1$, the restriction of $\widehat{\mu}$ to $a \boldsymbol{v}, a \in \mathbb{R}$, can be interpreted as the one-dimensional Fourier transform of the projected measure $\mu_{\boldsymbol{v}}$. Indeed for $\boldsymbol{R} \in \Pi_{u}$ we have $a \boldsymbol{v} \cdot \boldsymbol{R}=a u$ and we can write upon splitting the integral

$$
\widehat{\mu}(a \boldsymbol{v})=\int \mathrm{d} u \int_{\Pi_{u}} \mathrm{e}^{\mathrm{i} a u} \mu=\int \mathrm{d} u \mathrm{e}^{\mathrm{i} a u} \mu_{\boldsymbol{v}}(u)=\widehat{\mu}_{\boldsymbol{v}}(a) .
$$

Thus, the scattered amplitude in a fixed geometry $(\boldsymbol{k}, q)=a \boldsymbol{v}$ is simply linked to the Fourier transform of the projected measure:

$$
A(a \boldsymbol{k}, a q)=\widehat{\mu}_{\boldsymbol{v}}(a)
$$

with the following consequence:

Corollary 2.5. Under the conditions of theorem 2.3 we have for any vector $(\boldsymbol{k}, q) \in \mathbb{R}^{n+1}$

$$
\lim _{a \rightarrow \infty} \sup _{\inf } \frac{\mathbb{E}|A(a \boldsymbol{k}, a q)|^{2}}{\log (1 / a)}=2 n / \alpha \text {. }
$$

\subsection{Application to Kirchhoff scattering}

The theoretical results have obvious applications to Kirchhoff scattering as we shall make explicit. We call a variable geometry scheme a configuration where the source and the receiver are allowed to vary freely in space and a fixed geometry scheme a configuration where the position of the latter is fixed but the frequency can vary. The former case corresponds to $(\boldsymbol{k}, q)$ varying freely in $\mathbb{R}^{n+1}$ while the latter case corresponds to a set $(a \boldsymbol{k}, a q), a \in \mathbb{R}$, for a fixed direction $\boldsymbol{v}=(\boldsymbol{k}, q)$. We call $I(\boldsymbol{k}, q)=|A(\boldsymbol{k}, q)|^{2}$ the intensity. Note that no phase information is needed for this quantity, which makes it easier to assess in experiments than the full amplitude $A$. Moreover, we define the average intensity at maximum frequency $K$ :

$$
\tilde{I}(K)=\frac{1}{K^{n+1}} \int_{k^{2}+q^{2} \leqslant K^{2}} \mathrm{~d} \boldsymbol{k} \mathrm{d} q I(\boldsymbol{k}, q) .
$$

We then have the following results, which are immediate applications of the previous theorems.

Corollary 2.6. Let $h(\boldsymbol{r})$ be a random fractal surface given by a fractional Brownian field with self-similarity exponent $\alpha$. Then $\alpha$ can be recovered almost surely from the scattered intensity in the variable geometry scheme:

$$
\lim _{K \rightarrow \infty} \sup _{\inf } \frac{\log \tilde{I}(K)}{\log 1 / K}=n+1-\alpha / 2 \text { a.s. }
$$

In the fixed geometry scheme, $\alpha$ can be recovered from a characteristic set of surfaces through the scaling behaviour of the average intensity:

$$
\lim _{a \rightarrow \infty} \sup _{\inf } \frac{\log \tilde{\mathbb{E}} I(a \boldsymbol{k}, a q)}{\log 1 / a}=2 n / \alpha .
$$




\section{Proofs of the main theorems}

\subsection{Asymptotically self-similar Gaussian random surfaces}

We will prove the main theorems for a class of Gaussian random surfaces which include the fractional Brownian surfaces as particular case. Let $\mathcal{C}_{\alpha}$ be the class of centred Gaussian fields $h(\boldsymbol{r})$ on $\mathbb{R}^{n}$ satisfying the following properties:

- The increments $h\left(\boldsymbol{r}_{1}\right)-h\left(\boldsymbol{r}_{2}\right)$ are (wide sense) stationary and isotropic, with correlation

$$
C\left(\boldsymbol{r}_{1}, \boldsymbol{r}_{2}\right):=\mathbb{E} h\left(\boldsymbol{r}_{1}\right) h\left(\boldsymbol{r}_{2}\right)
$$

and structure function

$$
S\left(\boldsymbol{r}_{1}, \boldsymbol{r}_{2}\right)=: S\left(\left\|\boldsymbol{r}_{1}-\boldsymbol{r}_{2}\right\|\right)=\mathbb{E}\left|h\left(\boldsymbol{r}_{1}\right)-h\left(\boldsymbol{r}_{2}\right)\right|^{2} .
$$

- The structure function is asymptotically self-similar at small scales, that is

$$
S\left(\left\|\boldsymbol{r}_{1}-\boldsymbol{r}_{2}\right\|\right)=2 \sigma^{2}\left\|\boldsymbol{r}_{1}-\boldsymbol{r}_{2}\right\|^{\alpha} \varphi\left(\left\|\boldsymbol{r}_{1}-\boldsymbol{r}_{2}\right\|\right)
$$

for some constants $0<\alpha<2$ and $\sigma>0$ and a continuous positive bounded function $\varphi$ such that $\varphi(0)=1$.

- The first two derivatives of $\varphi$ satisfy:

$$
\left|\varphi^{\prime}(r)\right| \leqslant \text { const } \times\left|\frac{\varphi(r)}{r}\right| \quad\left|\varphi^{\prime \prime}(r)\right| \leqslant \text { const } \times\left|\frac{\varphi(r)}{r^{2}}\right| \quad r>0 .
$$

The parameter $\sigma$ in (3.16) is a vertical dilation parameter and the factor 2 is introduced for convenience. A finite non-zero value for $\varphi(0)$ expresses the asymptotic self-similarity of the surface at small scales. Finally, the requirement (3.17) on the derivatives of $\varphi$ is a nonoscillating condition for the correlation function. Isotropic fractional Brownian motion (fBm) on $\mathbb{R}^{n}$ is paradigmatic of the class $\mathcal{C}_{\alpha}$, and is obtained for $\varphi=1$. Stationary processes with covariance of the form,

$$
C\left(\boldsymbol{r}_{1}, \boldsymbol{r}_{2}\right)=F\left(\left\|\boldsymbol{r}_{1}-\boldsymbol{r}_{2}\right\|^{\alpha}\right)
$$

where $F$ is twice differentiable, are another instance of the class $\mathcal{C}_{\alpha}$ (a usual example is the Weilbulian covariance $\mathbb{E} h\left(\boldsymbol{r}_{1}\right) h\left(\boldsymbol{r}_{2}\right)=\exp \left(-\left\|\boldsymbol{r}_{1}-\boldsymbol{r}_{2}\right\|^{\alpha}\right)$. Another example can be found in the family of $1 / f$ processes, i.e. stationary processes whose power spectrum $\Gamma(\xi)$ has an asymptotic power-law: $\Gamma(\boldsymbol{\xi}) \sim\|\xi\|^{-\alpha-1},\|\xi\| \rightarrow \infty$.

\subsection{The graph measure}

Let again $\mu$ be the graph measure associated with a realization $h$ of a process in class $\mathcal{C}_{\alpha}$. We will prove the following theorem.

Theorem 3.1. Let $h$ be a version of a process in class $\mathcal{C}_{\alpha}$ over $\mathbb{R}^{n}$ with continuous trajectories. Let $\mu$ be the graph measure associated with each trajectory. Then the upper and lower correlation dimensions of $\mu$ are random variables, which almost surely are the following constants:

$$
d_{c}^{ \pm}[\mu]=n+1-\alpha / 2
$$

Proof. Let $X(\epsilon)$ be the correlation integral (2.2) associated with $\mu$. By definition of the measure $\mu$ we have ${ }^{4}$

$X(\epsilon)=\int_{\mathbb{R}^{n} \times \mathbb{R}^{n}} \mathrm{~d} \boldsymbol{r}^{2} \chi\left(\boldsymbol{r}_{1}\right) \chi\left(\boldsymbol{r}_{2}\right) H\left(\epsilon^{2}-\left(\left\|\boldsymbol{r}_{1}-\boldsymbol{r}_{2}\right\|^{2}+\left|h\left(\boldsymbol{r}_{1}\right)-h\left(\boldsymbol{r}_{2}\right)\right|^{2}\right)\right)$

4 Here and everywhere we will use the short-hand notation $\mathrm{d} \boldsymbol{r}^{m}:=\mathrm{d} \boldsymbol{r}_{1} . . \mathrm{d} \boldsymbol{r}_{m}$ for a multiple integral. 
and thus

$\mathbb{E} X(\epsilon)=\int_{\mathbb{R}^{n} \times \mathbb{R}^{n}} \mathrm{~d} \boldsymbol{r}^{2} \chi\left(\boldsymbol{r}_{1}\right) \chi\left(\boldsymbol{r}_{2}\right)$ Proba $\left[\left\|\boldsymbol{r}_{1}-\boldsymbol{r}_{2}\right\|^{2}+\left|h\left(\boldsymbol{r}_{1}\right)-h\left(\boldsymbol{r}_{2}\right)\right|^{2} \leqslant \epsilon^{2}\right]$.

Now the increment $h\left(\boldsymbol{r}_{1}\right)-h\left(\boldsymbol{r}_{2}\right)$ is a centred Gaussian process with p.d.f:

$p\left(\boldsymbol{r}_{1}, \boldsymbol{r}_{2} ; u\right):=p\left(\left\|\boldsymbol{r}_{1}-\boldsymbol{r}_{2}\right\| ; u\right)=\frac{1}{\sqrt{2 \pi S\left(\left\|\boldsymbol{r}_{1}-\boldsymbol{r}_{2}\right\|\right)}} \exp \left(-\frac{u^{2}}{2 S\left(\left\|\boldsymbol{r}_{1}-\boldsymbol{r}_{2}\right\|\right)}\right)$

and thus

$$
\mathbb{E} X(\epsilon)=\int \mathrm{d} \boldsymbol{r}^{2} H\left(\epsilon-\left\|\boldsymbol{r}_{1}-\boldsymbol{r}_{2}\right\|\right) \chi\left(\boldsymbol{r}_{1}\right) \chi\left(\boldsymbol{r}_{2}\right) U\left(\left\|\boldsymbol{r}_{1}-\boldsymbol{r}_{2}\right\| ; \epsilon\right)
$$

where we have introduced the kernel:

$$
U(r ; \epsilon)=\operatorname{Erf}\left(\sqrt{\frac{\epsilon^{2}-r^{2}}{2 S(r)}}\right)
$$

and the error function:

$$
\operatorname{Erf}(r):=\frac{2}{\sqrt{\pi}} \int_{0}^{r} \mathrm{~d} t \mathrm{e}^{-t^{2}} \quad r \geqslant 0 .
$$

In view of the inequality

$\chi\left(2\left(\boldsymbol{r}_{1}-\boldsymbol{r}_{2}\right)\right) \chi\left(2\left(\boldsymbol{r}_{1}+\boldsymbol{r}_{2}\right)\right) \leqslant \chi\left(\boldsymbol{r}_{1}\right) \chi\left(\boldsymbol{r}_{2}\right) \leqslant \chi\left(\frac{\boldsymbol{r}_{1}-\boldsymbol{r}_{2}}{2}\right) \chi\left(\frac{\boldsymbol{r}_{1}+\boldsymbol{r}_{2}}{2}\right)$

this implies:

$\mathbb{E} X(\epsilon) \geqslant \int \mathrm{d} \boldsymbol{r}^{2} H\left(\epsilon-\left\|\boldsymbol{r}_{1}-\boldsymbol{r}_{2}\right\|\right) \chi\left(2\left(\boldsymbol{r}_{1}-\boldsymbol{r}_{2}\right)\right) \chi\left(2\left(\boldsymbol{r}_{1}+\boldsymbol{r}_{2}\right)\right) U\left(\left\|\boldsymbol{r}_{1}-\boldsymbol{r}_{2}\right\| ; \epsilon\right)$
$\mathbb{E} X(\epsilon) \leqslant \int \mathrm{d} \boldsymbol{r}^{2} H\left(\epsilon-\left\|\boldsymbol{r}_{1}-\boldsymbol{r}_{2}\right\|\right) \chi\left(\frac{\boldsymbol{r}_{1}-\boldsymbol{r}_{2}}{2}\right) \chi\left(\frac{\boldsymbol{r}_{1}+\boldsymbol{r}_{2}}{2}\right) U\left(\left\|\boldsymbol{r}_{1}-\boldsymbol{r}_{2}\right\| ; \epsilon\right)$.

With the change of variables $\left(\boldsymbol{r}_{1}, \boldsymbol{r}_{2}\right) \rightarrow\left(\boldsymbol{r}_{1}-\boldsymbol{r}_{2}, \boldsymbol{r}_{1}+\boldsymbol{r}_{2}\right)$ we obtain for $\epsilon<1 / 2\left(s_{n}\right.$ is the surface of the unit sphere in $\mathbb{R}^{n}$ ):

$$
\begin{aligned}
\mathbb{E} X(\epsilon) & \leqslant \int \mathrm{d} \boldsymbol{r} H(\epsilon-\|\boldsymbol{r}\|) U(\|\boldsymbol{r}\| ; \epsilon) \chi(\boldsymbol{r} / 2)=s_{n} \epsilon^{n} \int_{0}^{1} \mathrm{~d} r r^{n-1} U(\epsilon r ; \epsilon) \\
& =s_{n} \epsilon^{n} \int_{0}^{1} \mathrm{~d} r r^{n-1} \operatorname{Erf}\left(\epsilon^{1-\alpha / 2} \sqrt{\frac{1-r^{2}}{2 r^{\alpha} \varphi(\epsilon r)}}\right) \\
& \leqslant s_{n} \epsilon^{n} \int_{0}^{1} \mathrm{~d} r r^{n-1} \operatorname{Erf}\left(\epsilon^{1-\alpha / 2} \sqrt{\frac{1-r^{2}}{2 \varphi_{1} r^{\alpha}}}\right)
\end{aligned}
$$

and similarly

$$
\mathbb{E} X(\epsilon) \geqslant s_{n} 4^{-n} \epsilon^{n} \int_{0}^{1} \mathrm{~d} r r^{n-1} \operatorname{Erf}\left(\epsilon^{1-\alpha / 2} \sqrt{\frac{1-r^{2}}{2 \varphi_{2} r^{\alpha}}}\right)
$$

where $\varphi_{1}=\min _{[0,1]} \varphi(t)>0$ and $\varphi_{2}=\max _{[0,1]} \varphi(t)>0$. From the global estimation $\operatorname{Erf}(x)=2 / \sqrt{\pi}+O\left(x^{3}\right)$ we obtain the asymptotic behaviour:

$$
\mathbb{E} X(\epsilon) \sim \epsilon^{n+1-\alpha / 2} \text {. }
$$


Next we show that the standard deviation decreases faster than the mean. From (3.18) we have

$$
\begin{aligned}
& (\mathbb{E} X(\epsilon))^{2}=\int \mathrm{d} \boldsymbol{r}^{4} \chi\left(\boldsymbol{r}_{1}\right) \chi\left(\boldsymbol{r}_{2}\right) \chi\left(\boldsymbol{r}_{3}\right) \chi\left(\boldsymbol{r}_{4}\right) U\left(\left\|\boldsymbol{r}_{1}-\boldsymbol{r}_{2}\right\| ; \epsilon\right) U\left(\left\|\boldsymbol{r}_{3}-\boldsymbol{r}_{4}\right\| ; \epsilon\right) \\
& \mathbb{E} X^{2}(\epsilon)=\int \mathrm{d} \boldsymbol{r}^{4} \chi\left(\boldsymbol{r}_{1}\right) \chi\left(\boldsymbol{r}_{2}\right) \chi\left(\boldsymbol{r}_{3}\right) \chi\left(\boldsymbol{r}_{4}\right) U\left(\boldsymbol{r}_{1}, \boldsymbol{r}_{2}, \boldsymbol{r}_{3}, \boldsymbol{r}_{4} ; \epsilon\right)
\end{aligned}
$$

where we have introduced the kernel $U\left(\boldsymbol{r}_{1}, \boldsymbol{r}_{2}, \boldsymbol{r}_{3}, \boldsymbol{r}_{4} ; \epsilon\right)$ :

Proba $\left[\left\|\boldsymbol{r}_{1}-\boldsymbol{r}_{2}\right\|^{2}+\left|h\left(\boldsymbol{r}_{1}\right)-h\left(\boldsymbol{r}_{2}\right)\right|^{2} \leqslant \epsilon^{2},\left\|\boldsymbol{r}_{3}-\boldsymbol{r}_{4}\right\|^{2}+\left|h\left(\boldsymbol{r}_{3}\right)-h\left(\boldsymbol{r}_{4}\right)\right|^{2} \leqslant \epsilon^{2}\right]$.

Note that the effective domain of integration in the above integrals is reduced to a region $\left\|\boldsymbol{r}_{1}-\boldsymbol{r}_{2}\right\|<\epsilon,\left\|\boldsymbol{r}_{3}-\boldsymbol{r}_{4}\right\|<\epsilon$, as follows clearly from the definition of the kernel $U$.

To estimate the kernel we need the p.d.f of the Gaussian vector $\left(u=h\left(\boldsymbol{r}_{1}\right)-h\left(\boldsymbol{r}_{2}\right), u^{\prime}=\right.$ $\left.h\left(\boldsymbol{r}_{3}\right)-h\left(\boldsymbol{r}_{4}\right)\right)$ :

$$
p\left(\boldsymbol{r}_{1}, \boldsymbol{r}_{2}, \boldsymbol{r}_{3}, \boldsymbol{r}_{4} ; u, u^{\prime}\right)=\frac{1}{2 \pi \Delta} \exp \left(-\frac{1}{2 \Delta^{2}}\left(u^{2} s^{\prime 2}+u^{\prime 2} s^{2}-2 \rho s s^{\prime} u u^{\prime}\right)\right)
$$

with simplified notation:

$$
\begin{aligned}
& s^{2}=S\left(\left\|\boldsymbol{r}_{1}-\boldsymbol{r}_{2}\right\|\right) \quad s^{\prime 2}=S\left(\left\|\boldsymbol{r}_{3}-\boldsymbol{r}_{4}\right\|\right) \\
& s s^{\prime} \rho=\mathbb{E}\left[h\left(\boldsymbol{r}_{1}\right)-h\left(\boldsymbol{r}_{2}\right)\right]\left[h\left(\boldsymbol{r}_{3}\right)-h\left(\boldsymbol{r}_{4}\right)\right] \quad \Delta^{2}=s^{2} s^{\prime 2}\left(1-\rho^{2}\right) .
\end{aligned}
$$

Then by definition of (3.25)

$$
U\left(\boldsymbol{r}_{1}, \boldsymbol{r}_{2}, \boldsymbol{r}_{3}, \boldsymbol{r}_{4} ; \epsilon\right)=\int_{-\sqrt{\epsilon^{2}-\left\|\boldsymbol{r}_{1}-\boldsymbol{r}_{2}\right\|^{2}}}^{+\sqrt{\epsilon^{2}-\left\|\boldsymbol{r}_{1}-\boldsymbol{r}_{2}\right\|^{2}}} \mathrm{~d} u \int_{-\sqrt{\epsilon^{2}-\left\|\boldsymbol{r}_{3}-\boldsymbol{r}_{4}\right\|^{2}}}^{+\sqrt{\epsilon^{2}-\left\|\boldsymbol{r}_{3}-\boldsymbol{r}_{4}\right\|^{2}}} \mathrm{~d} u^{\prime} p\left(\boldsymbol{r}_{1}, \boldsymbol{r}_{2}, \boldsymbol{r}_{3}, \boldsymbol{r}_{4}, u, u^{\prime}\right) .
$$

Now the p.d.f can be factorized as

$$
p\left(\boldsymbol{r}_{1}, \boldsymbol{r}_{2}, \boldsymbol{r}_{3}, \boldsymbol{r}_{4} ; u, u^{\prime}\right)=\frac{1}{\sqrt{2} \pi} \frac{s}{\Delta} p\left(\boldsymbol{r}_{1}, \boldsymbol{r}_{2} ; u\right) \exp \left(-\frac{1}{2 \Delta^{2}}\left(s u^{\prime}-\rho s^{\prime} u\right)^{2}\right)
$$

where $p\left(\boldsymbol{r}_{1}, \boldsymbol{r}_{2} ; u\right)$ is the p.d.f (3.19), so the kernel $U\left(\boldsymbol{r}_{1}, \boldsymbol{r}_{2}, \boldsymbol{r}_{3}, \boldsymbol{r}_{4} ; \epsilon\right)$ may be rewritten as

$$
\int_{-\sqrt{\epsilon^{2}-\left\|\boldsymbol{r}_{1}-\boldsymbol{r}_{2}\right\|^{2}}}^{+\sqrt{\epsilon^{2}-\left\|\boldsymbol{r}_{1}-\boldsymbol{r}_{2}\right\|^{2}}} \mathrm{~d} u \frac{1}{\sqrt{2} \pi} \frac{s}{\Delta} p\left(\left\|\boldsymbol{r}_{1}-\boldsymbol{r}_{2}\right\| ; u\right) \int_{-\sqrt{\epsilon^{2}-\left\|\boldsymbol{r}_{3}-\boldsymbol{r}_{4}\right\|^{2}}}^{+\sqrt{\epsilon^{2}-\left\|\boldsymbol{r}_{3}-\boldsymbol{r}_{4}\right\|^{2}}} \mathrm{~d} u^{\prime} \exp \left(-\frac{1}{2 \Delta^{2}}\left(s u^{\prime}-\rho s^{\prime} u\right)^{2}\right)
$$

which in view of the inequality in lemma 4.2 yields

$$
U\left(\boldsymbol{r}_{1}, \boldsymbol{r}_{2}, \boldsymbol{r}_{3}, \boldsymbol{r}_{4} ; \epsilon\right) \leqslant U\left(\left\|\boldsymbol{r}_{1}-\boldsymbol{r}_{2}\right\| ; \epsilon\right) \tilde{U}\left(\boldsymbol{r}_{1}, \boldsymbol{r}_{2}, \boldsymbol{r}_{3}, \boldsymbol{r}_{4} ; \epsilon\right)
$$

with a modified kernel:

$$
\tilde{U}\left(\boldsymbol{r}_{1}, \boldsymbol{r}_{2}, \boldsymbol{r}_{3}, \boldsymbol{r}_{4} ; \epsilon\right)=\operatorname{Erf}\left(\sqrt{\frac{s^{2}\left(\epsilon^{2}-\left\|\boldsymbol{r}_{3}-\boldsymbol{r}_{4}\right\|^{2}\right)}{2 \Delta^{2}}}\right) .
$$

Since

$$
\begin{aligned}
\operatorname{Var} X(\epsilon)=\int & \mathrm{d} \boldsymbol{r}^{4}\left[U\left(\boldsymbol{r}_{1}, \boldsymbol{r}_{2}, \boldsymbol{r}_{3}, \boldsymbol{r}_{4} ; \epsilon\right)\right. \\
& \left.-U\left(\left\|\boldsymbol{r}_{1}-\boldsymbol{r}_{2}\right\| ; \epsilon\right) U\left(\left\|\boldsymbol{r}_{3}-\boldsymbol{r}_{4}\right\| ; \epsilon\right)\right] \chi\left(\boldsymbol{r}_{1}\right) \chi\left(\boldsymbol{r}_{2}\right) \chi\left(\boldsymbol{r}_{3}\right) \chi\left(\boldsymbol{r}_{4}\right)
\end{aligned}
$$

we thus have

$$
\begin{aligned}
\operatorname{Var} X(\epsilon) \leqslant \int & \mathrm{d} \boldsymbol{r}^{4} U\left(\left\|\boldsymbol{r}_{1}-\boldsymbol{r}_{2}\right\| ; \epsilon\right) U\left(\left\|\boldsymbol{r}_{3}-\boldsymbol{r}_{4}\right\| ; \epsilon\right) \\
& \times \Delta U\left(\boldsymbol{r}_{1}, \boldsymbol{r}_{2}, \boldsymbol{r}_{3}, \boldsymbol{r}_{4} ; \epsilon\right) \chi\left(\boldsymbol{r}_{1}\right) \chi\left(\boldsymbol{r}_{2}\right) \chi\left(\boldsymbol{r}_{3}\right) \chi\left(\boldsymbol{r}_{4}\right)
\end{aligned}
$$


with

$$
\Delta U\left(\boldsymbol{r}_{1}, \boldsymbol{r}_{2}, \boldsymbol{r}_{3}, \boldsymbol{r}_{4} ; \epsilon\right)=\frac{\tilde{U}\left(\boldsymbol{r}_{1}, \boldsymbol{r}_{2}, \boldsymbol{r}_{3}, \boldsymbol{r}_{4} ; \epsilon\right)-U\left(\left\|\boldsymbol{r}_{3}-\boldsymbol{r}_{4}\right\| ; \epsilon\right)}{U\left(\left\|\boldsymbol{r}_{3}-\boldsymbol{r}_{4}\right\| ; \epsilon\right)} .
$$

To estimate this quantity we introduce the function:

$$
f(\tau):=\operatorname{Erf}\left(\sqrt{\frac{\tau\left(\epsilon^{2}-\left\|\boldsymbol{r}_{3}-\boldsymbol{r}_{4}\right\|^{2}\right)}{2}}\right)
$$

for which we observe that

$$
f(s / \Delta)=\tilde{U}\left(\boldsymbol{r}_{1}, \boldsymbol{r}_{2}, \boldsymbol{r}_{3}, \boldsymbol{r}_{4} ; \epsilon\right) \geqslant f\left(1 / s^{\prime}\right)=U\left(\boldsymbol{r}_{3}, \boldsymbol{r}_{4} ; \epsilon\right) .
$$

Applying the mean value theorem to $f$ and noting that it has a decreasing derivative we obtain the following estimation:

$$
\Delta U\left(\boldsymbol{r}_{1}, \boldsymbol{r}_{2}, \boldsymbol{r}_{3}, \boldsymbol{r}_{4} ; \epsilon\right) \leqslant\left|\frac{s s^{\prime}}{\Delta}-1\right| \sup _{t>0} \frac{t \mathrm{e}^{-t^{2}}}{\operatorname{Erf}(t)} \leqslant \text { const } \times \frac{\rho}{1-\rho} .
$$

Since $\rho$ is not bounded away from 1 we have to distinguish two domains. For any $0<\beta<1$ we define two complementary domains $\mathcal{D}_{\epsilon}$ and $\mathcal{D}_{\epsilon}^{c}$ by

$\mathcal{D}_{\epsilon}=\left\{\left(\boldsymbol{r}_{1}, \boldsymbol{r}_{2}, \boldsymbol{r}_{3}, \boldsymbol{r}_{4}\right) \in\left(\mathbb{R}^{n}\right)^{4}:\left\|\boldsymbol{r}_{1}-\boldsymbol{r}_{2}\right\|<\epsilon,\left\|\boldsymbol{r}_{3}-\boldsymbol{r}_{4}\right\|<\epsilon,\left\|\boldsymbol{r}_{1}-\boldsymbol{r}_{3}\right\|<\epsilon^{\beta}\right\}$

$\mathcal{D}_{\epsilon}^{c}=\left\{\left(\boldsymbol{r}_{1}, \boldsymbol{r}_{2}, \boldsymbol{r}_{3}, \boldsymbol{r}_{4}\right) \in\left(\mathbb{R}^{n}\right)^{4}:\left\|\boldsymbol{r}_{1}-\boldsymbol{r}_{2}\right\|<\epsilon,\left\|\boldsymbol{r}_{3}-\boldsymbol{r}_{4}\right\|<\epsilon,\left\|\boldsymbol{r}_{1}-\boldsymbol{r}_{3}\right\|>\epsilon^{\beta}\right\}$.

On the domain $\mathcal{D}_{\epsilon}^{c}$ we have $\frac{\left\|r_{1}-r_{2}\right\|+\left\|r_{3}-r_{4}\right\|}{\left\|r_{1}-r_{3}\right\|} \leqslant 2 \epsilon^{1-\beta}<1$ for $\epsilon$ small enough, so we may apply the lemma 4.1 in the appendix:

$$
\rho s s^{\prime} \leqslant \text { const } \times\left\|\boldsymbol{r}_{1}-\boldsymbol{r}_{2}\right\|\left\|\boldsymbol{r}_{3}-\boldsymbol{r}_{4}\right\|\left\|\boldsymbol{r}_{1}-\boldsymbol{r}_{3}\right\|^{-(2-\alpha)}
$$

which in particular implies

$$
\rho \leqslant \text { const } \times \epsilon^{(2-\alpha)(1-\beta)}
$$

with a positive exponent. On $\mathcal{D}_{\epsilon}$ we use the simple fact that $U\left(\left\|\boldsymbol{r}_{3}-\boldsymbol{r}_{4}\right\|\right) \Delta U\left(\boldsymbol{r}_{1}, \boldsymbol{r}_{2}\right.$, $\left.\boldsymbol{r}_{3}, \boldsymbol{r}_{4} ; \epsilon\right) \leqslant \tilde{U}\left(\boldsymbol{r}_{1}, \boldsymbol{r}_{2}, \boldsymbol{r}_{3}, \boldsymbol{r}_{4} ; \epsilon\right) \leqslant 1$, together with the uniform estimation:

$$
\int \mathrm{d} \boldsymbol{r}^{2} H\left(\epsilon-\left\|\boldsymbol{r}_{3}-\boldsymbol{r}_{4}\right\|\right) H\left(\epsilon^{\beta}-\left\|\boldsymbol{r}_{1}-\boldsymbol{r}_{3}\right\|\right) \chi\left(\boldsymbol{r}_{3}\right) \chi\left(\boldsymbol{r}_{4}\right) \leqslant \text { const } \times \epsilon^{n(1+\beta)} \text {. }
$$

Altogether this yields:

$\operatorname{Var} X(\epsilon) \leqslant$ const $\times \epsilon^{n(1+\beta)} \mathbb{E} X(\epsilon)+$ const $\times \epsilon^{(2-\alpha)(1-\beta)} \mathbb{E}^{2} X(\epsilon) \leqslant$ const $\times \epsilon^{\nu} \mathbb{E}^{2} X(\epsilon)$

with $v=\min \{n \beta+\alpha / 2-1,(2-\alpha)(1-\beta)\}$. Now we can chose the optimal $\beta$. A direct computation shows that the optimal exponent is

$$
\sup _{0<\beta<1} v=\frac{(2 n-2+\alpha)(1-\alpha / 2)}{n+2-\alpha}>0 .
$$

We conclude with a standard argument using Tchebyshev inequality and Borel-Cantelli lemma that $X(\epsilon) \sim \mathbb{E} X(\epsilon)$ a.s. as $\epsilon \rightarrow 0$. In view of (3.24) this completes the proof.

\subsection{The projected measure}

Let us now prove theorem 2.3 for asymptotically self-similar processes.

Theorem 3.2. Let h be a version of a process in class $\mathcal{C}_{\alpha}$ over $\mathbb{R}^{n}$ with continuous trajectories. For fixed $\boldsymbol{v}(\boldsymbol{k}, q) \in \mathbb{R}^{n+1}$ let $\mu_{\boldsymbol{v}}$ be the projection of the graph measure along $\boldsymbol{v}^{\perp}$ associated with each trajectory. Then we have

$$
\mathbb{E} d_{c}^{ \pm}\left[\mu_{v}\right]=1 \text {. }
$$


Proof. The proof is very similar to that of theorem 3.1 so we will make it short. Let $X(\epsilon)$ be the correlation integral (2.2) associated with $\mu_{\boldsymbol{v}}$. To pick a point uniformly according to the measure $\mu_{\boldsymbol{v}}$, we pick a point $\boldsymbol{r}$ uniformly on the unit ball of $\mathbb{R}^{n}$ and form $\boldsymbol{k} \cdot \boldsymbol{r}+q h(\boldsymbol{r})$. We thus have

$X(\epsilon)=\int_{\mathbb{R}^{n} \times \mathbb{R}^{n}} \mathrm{~d} \boldsymbol{r}^{2} \chi\left(\boldsymbol{r}_{1}\right) \chi\left(\boldsymbol{r}_{2}\right) H\left(\epsilon-\left|\boldsymbol{k} \cdot\left(\boldsymbol{r}_{1}-\boldsymbol{r}_{2}\right)+q\left(h\left(\boldsymbol{r}_{1}\right)-h\left(\boldsymbol{r}_{2}\right)\right)\right|\right)$.

Similar calculations as previously yield

$\mathbb{E} X(\epsilon)=\int \mathrm{d} \boldsymbol{r}^{2} \chi\left(\boldsymbol{r}_{1}\right) \chi\left(\boldsymbol{r}_{2}\right)\left[\operatorname{Erf}\left(\frac{\epsilon-\boldsymbol{k} \cdot\left(\boldsymbol{r}_{1}-\boldsymbol{r}_{2}\right)}{\sqrt{2 S\left(\boldsymbol{r}_{1}-\boldsymbol{r}_{2}\right)}}\right)-\operatorname{Erf}\left(\frac{-\epsilon-\boldsymbol{k} \cdot\left(\boldsymbol{r}_{1}-\boldsymbol{r}_{2}\right)}{\sqrt{2 S\left(\boldsymbol{r}_{1}-\boldsymbol{r}_{2}\right)}}\right)\right]$.

It is easy to check that the above function is differentiable with respect to $\epsilon>0$, with a finite derivative in zero. This shows that

$$
\mathbb{E} X(\epsilon) \sim \epsilon \quad \epsilon \rightarrow 0 .
$$

Now from Jensen's inequality we have

$$
\log \mathbb{E} X(\epsilon) \geqslant \mathbb{E} \log X(\epsilon)
$$

which, combined with (3.28), implies $\mathbb{E} d_{c}^{ \pm}\left[\mu_{v}\right] \geqslant 1$. Since 1 is the maximum reachable value for a one-dimensional probability measure, this implies the stated result.

Remark. A correlation integral similar to (3.27) has been considered in [18] for $\mathbb{R}^{d}$-valued $(d \geqslant 1)$ random processes over $\mathbb{R}$ and for $k=0$. Specializing the results of this paper to $d=1$ and to fractional Brownian motion one obtains $d_{c}=1$ almost surely.

Theorem 3.3. Under the same conditions as theorem 2.3 let $\mu_{v}$ be the projection of the graph measure perpendicular to a direction $\boldsymbol{v}=(\boldsymbol{k}, q)$. Then we have

$$
\lim _{a \rightarrow \infty} \sup \frac{\mathbb{E}\left|\widehat{\mu_{v}}(a)\right|^{2}}{\log (1 / a)}=2 n / \alpha .
$$

As a consequence, the projected measure has a non-trivial wavelet correlation dimension in the sense that:

$$
\mathbb{E} d_{w}^{ \pm}\left[\mu_{v}\right] \geqslant n(2 / \alpha-1)
$$

Proof. To establish the result we will use the relation $\widehat{\mu_{\boldsymbol{v}}}(a)=A(a \boldsymbol{k}, a q)$. Let us estimate $\mathbb{E} I(a \boldsymbol{k}, a q)$ with

$$
I(\boldsymbol{k}, q):=|A(\boldsymbol{k}, q)|^{2}=\int_{\mathbb{R}^{n} \times \mathbb{R}^{n}} \mathrm{~d} \boldsymbol{r}^{2} \chi\left(\boldsymbol{r}_{1}\right) \chi\left(\boldsymbol{r}_{2}\right) \mathrm{e}^{\mathrm{i} \boldsymbol{k} \cdot\left(\boldsymbol{r}_{1}-\boldsymbol{r}_{2}\right)+\mathrm{i} q\left(h\left(\boldsymbol{r}_{1}\right)-h\left(\boldsymbol{r}_{2}\right)\right)} .
$$

The mathematical expectation can be expressed using the characteristic function of the increments:

$$
\mathbb{E} \exp \left(\mathrm{i} q\left[h\left(\boldsymbol{r}_{1}\right)-h\left(\boldsymbol{r}_{2}\right)\right]\right)=\exp \left(-\frac{q^{2}}{2} S\left(\boldsymbol{r}_{1}-\boldsymbol{r}_{2}\right)\right)
$$

leading to

$\mathbb{E} I(a \boldsymbol{k}, a q)=\int_{\mathbb{R}^{n} \times \mathbb{R}^{n}} \mathrm{~d} \boldsymbol{r}^{2} \mathrm{e}^{\mathrm{i} a \boldsymbol{k} \cdot\left(\boldsymbol{r}_{1}-\boldsymbol{r}_{2}\right)} \exp \left(-\frac{a^{2} q^{2}}{2} S\left(\boldsymbol{r}_{1}-\boldsymbol{r}_{2}\right)\right) \chi\left(\boldsymbol{r}_{1}\right) \chi\left(\boldsymbol{r}_{2}\right)$.

Let us decompose this expression in

$$
\begin{aligned}
\mathbb{E} I(a \boldsymbol{k}, a q)= & \mathbb{E} I(\mathbf{0}, a q)+\int_{\mathbb{R}^{n} \times \mathbb{R}^{n}} \mathrm{~d} \boldsymbol{r}^{2}\left(\mathrm{e}^{\mathrm{i} a \boldsymbol{k} \cdot\left(\boldsymbol{r}_{1}-\boldsymbol{r}_{2}\right)}-1\right) \\
& \times \exp \left(-\frac{a^{2} q^{2}}{2} S\left(\boldsymbol{r}_{1}-\boldsymbol{r}_{2}\right)\right) \chi\left(\boldsymbol{r}_{1}\right) \chi\left(\boldsymbol{r}_{2}\right)
\end{aligned}
$$


and start with the evaluation of the first term on the right-hand side. Using (3.23) again and performing the change of variables $\left(\boldsymbol{r}_{1}, \boldsymbol{r}_{2}\right) \rightarrow\left(\boldsymbol{r}_{1}-\boldsymbol{r}_{2}, \boldsymbol{r}_{1}+\boldsymbol{r}_{2}\right)$ we obtain

$4^{-n} \int_{\mathbb{R}^{n}} \mathrm{~d} \boldsymbol{r} \exp \left(-\frac{a^{2} q^{2}}{2} S(\boldsymbol{r})\right) \chi(2 \boldsymbol{r}) \leqslant \mathbb{E} I(\mathbf{0}, a q) \leqslant \int_{\mathbb{R}^{n}} \mathrm{~d} \boldsymbol{r} \exp \left(-\frac{a^{2} q^{2}}{2} S(\boldsymbol{r})\right) \chi(\boldsymbol{r} / 2)$.

The above integrands are radial functions and thus we also have

$$
\begin{array}{r}
4^{-n} s_{n} \int_{0}^{\infty} r^{n-1} \exp \left(-\frac{a^{2} q^{2}}{2} S(r)\right) \chi(2 r) \leqslant \mathbb{E} I(\mathbf{0}, a q) \\
\leqslant s_{n} \int_{0}^{\infty} r^{n-1} \exp \left(-\frac{a^{2} q^{2}}{2} S(r)\right) \chi(r / 2)
\end{array}
$$

where $s_{n}$ is the surface of the unit sphere in $\mathbb{R}^{n}$. Using the asymptotic behaviour $S(r) \sim r^{\alpha}$ we obtain after straightforward calculations:

$$
\mathbb{E} I(\mathbf{0}, a q) \sim a^{-2 n / \alpha} .
$$

The contribution of the remaining term can be estimated with the same change of variables as previously, leading to

$$
a^{2 n / \alpha}|\mathbb{E} I(a \boldsymbol{k}, a q)-\mathbb{E} I(\mathbf{0}, a q)| \leqslant a^{1-2 / \alpha} \quad a \rightarrow \infty .
$$

Since $2 / \alpha>1$, this shows (3.29). The second part of theorem is a consequence of definition (2.11) and Jensen's inequality $\log \mathbb{E} \int I(a \boldsymbol{k}, a q) \geqslant \mathbb{E} \log \int I(a \boldsymbol{k}, a q)$.

\section{Appendix. Technical lemmas}

Lemma A.1. Let $h$ be a process in the class $\mathcal{C}_{\alpha}$ and $0<\theta<1$. Then for $\left\|\boldsymbol{r}_{1}-\boldsymbol{r}_{2}\right\|+\left\|\boldsymbol{r}_{3}-\boldsymbol{r}_{4}\right\| \leqslant$ $\theta\left\|\boldsymbol{r}_{1}-\boldsymbol{r}_{3}\right\|$ we have

$\left|\mathbb{E}\left(h\left(\boldsymbol{r}_{1}\right)-h\left(\boldsymbol{r}_{2}\right)\right)\left(h\left(\boldsymbol{r}_{3}\right)-h\left(\boldsymbol{r}_{4}\right)\right)\right| \leqslant \mathrm{const} \times\left\|\boldsymbol{r}_{1}-\boldsymbol{r}_{2}\right\|\left\|\boldsymbol{r}_{3}-\boldsymbol{r}_{4}\right\|\left\|\boldsymbol{r}_{1}-\boldsymbol{r}_{3}\right\|^{\alpha-2}$.

Proof. Introducing the correlation function we can write:

$$
\begin{aligned}
\mathbb{E}\left(h\left(\boldsymbol{r}_{1}\right)-h\left(\boldsymbol{r}_{2}\right)\right)\left(h\left(\boldsymbol{r}_{3}\right)-h\left(\boldsymbol{r}_{4}\right)\right) & =C\left(\boldsymbol{r}_{1}, \boldsymbol{r}_{3}\right)+C\left(\boldsymbol{r}_{2}, \boldsymbol{r}_{4}\right)-C\left(\boldsymbol{r}_{1}, \boldsymbol{r}_{4}\right)-C\left(\boldsymbol{r}_{2}, \boldsymbol{r}_{3}\right) \\
& =\int_{0}^{\left\|\boldsymbol{r}_{2}-\boldsymbol{r}_{1}\right\|} \mathrm{d} u \int_{0}^{\left\|\boldsymbol{r}_{3}-\boldsymbol{r}_{4}\right\|} \mathrm{d} v \partial_{u} \partial_{v} C\left(\boldsymbol{r}_{1}+u \boldsymbol{a}, \boldsymbol{r}_{3}+v \boldsymbol{b}\right)
\end{aligned}
$$

with

$$
a=\frac{\boldsymbol{r}_{2}-\boldsymbol{r}_{1}}{\left\|\boldsymbol{r}_{2}-\boldsymbol{r}_{1}\right\|} \quad \boldsymbol{b}=\frac{\boldsymbol{r}_{3}-\boldsymbol{r}_{4}}{\left\|\boldsymbol{r}_{3}-\boldsymbol{r}_{4}\right\|}
$$

Now

$\partial_{u} \partial_{v} C\left(\boldsymbol{r}_{1}+u \boldsymbol{a}, \boldsymbol{r}_{3}+v \boldsymbol{b}\right)=\partial_{u} \partial_{v} S(R(u, v))=\left(\partial_{u} \partial_{v} R\right) S^{\prime}(R)+\left(\partial_{u} R \partial_{v} R\right) S^{\prime \prime}(R)$

with $R(u, v)=\left\|\boldsymbol{r}_{1}-\boldsymbol{r}_{3}+u \boldsymbol{a}-v \boldsymbol{b}\right\|$, and a straightforward calculation shows that

$R\left|\begin{array}{l}\partial_{u} \\ \partial_{v}\end{array}(u, v)\right| \leqslant|u|+|v|+\left\|\boldsymbol{r}_{1}-\boldsymbol{r}_{3}\right\| \quad R\left|\partial_{u} \partial_{v} R(u, v)\right| \leqslant 1+\frac{|u|+|v|+\left\|\boldsymbol{r}_{1}-\boldsymbol{r}_{3}\right\|}{R^{2}(u, v)}$.

By hypothesis $\|u \boldsymbol{a}-v \boldsymbol{b}\| \leqslant|u|+|v| \leqslant \theta\left\|\boldsymbol{r}_{1}-\boldsymbol{r}_{3}\right\|$ which entails

$$
R \sim\left\|\boldsymbol{r}_{1}-\boldsymbol{r}_{3}\right\| \quad \partial_{u} R \sim 1 \quad \partial_{v} R \sim 1 \quad \partial_{u} \partial_{v} R \sim 1 /\left\|\boldsymbol{r}_{1}-\boldsymbol{r}_{3}\right\| .
$$

On the other hand it follows from (3.17) that

$$
\left|S^{\prime}(r)\right| \leqslant \text { const } \times r^{\alpha-1} \quad\left|S^{\prime \prime}(r)\right| \leqslant \text { const } \times r^{\alpha-2}
$$


and thus

$$
\left|\partial_{u} \partial_{v} C\left(\boldsymbol{r}_{1}+u \boldsymbol{a}, \boldsymbol{r}_{3}+v \boldsymbol{b}\right)\right| \leqslant \text { const } \times\left\|\boldsymbol{r}_{1}-\boldsymbol{r}_{3}\right\|^{\alpha-2} .
$$

The conclusion follows from the mean value theorem.

Lemma A.2. For all $a>0$ and $b$ in $\mathbb{R}$ the following inequality is satisfied:

$$
F(a, b):=\frac{2}{\sqrt{\pi}} \int_{-a}^{a} \exp \left(-(t-b)^{2}\right) \mathrm{d} t \leqslant 2 \operatorname{Erf}(a):=\frac{4}{\sqrt{\pi}} \int_{0}^{a} \exp \left(-t^{2}\right) \mathrm{d} t .
$$

Proof. Consider two cases. First suppose $|b|<a$. Then by a simple change of variables:

$$
F(a, b)=\operatorname{Erf}(a-b)+\operatorname{Erf}(a+b) \leqslant 2 \operatorname{Erf}(a)
$$

where the concavity of the error function on $\mathbb{R}^{+}$has been invoked for the last inequality. In the alternative case $|b| \geqslant a$ we have

$$
F(a, b)=\operatorname{Erf}(|b|+a)-\operatorname{Erf}(|b|-a) .
$$

Now the function $x \mapsto f_{a}(x)=\operatorname{Erf}(x+a)-\operatorname{Erf}(x-a)$ has a negative derivative on $[a,+\infty)$ and therefore reaches its maximum at $x=a$. Thus

$$
\sup _{|b| \geqslant a} F(a, b)=\operatorname{Erf}(2 a) \leqslant 2 \operatorname{Erf}(a) .
$$

This proves the lemma.

\section{References}

[1] Falconer K 1990 Fractal Geometry: Mathematical Foundations and Applications (New York: Wiley)

[2] Korvin G 1992 Fractal Models in the Earth Sciences (Amsterdam: Elsevier)

[3] Pfeifer P 1988 Chemistry and Physics of Solid Surfaces VII (Berlin: Springer) pp 283-303

[4] Beckmann P and Spizzichino A 1963 The Scattering of Electromagnetic Waves from Rough Surfaces (Oxford: Pergamon)

[5] Yordanov O I and Ivanova K 1994 Kirchhoff diffractals J. Phys. A: Math. Gen. 27 5979-93

[6] Jaggard D L and Sun X 1990 Scattering from fractally corrugated surfaces J. Opt. Soc. Am. 7 1131-9

[7] Franceschetti G, Iodice A, Migliaccio M and Riccio D 1999 Scattering from natural rough surfaces modeled by fractional Brownian motion IEEE Trans. Antennas Propag. 47 1405-15

[8] Berizzi F and Dalle-Mese E 1999 Fractal analysis of the signal scattered from the sea surface IEEE Trans. Antennas Propag. 47 324-38

[9] Chen J, Lo T K L, Leung H and Litva J 1996 The use of fractals for modeling em waves scattering from rough sea surfaces IEEE Trans. Geosci. Remote Sens. 34 966-72

[10] Berry M V 1981 Diffractal echoes J. Phys. A: Math. Gen. 14 3101-10

[11] Simonsen D, Vandembroucq I and Roux S 2001 Electromagnetic wave scattering from conducting self-affine surfaces: an analytic and numerical study J. Opt. Soc. Am. A 18 1101-11

[12] Guérin C A and Saillard M 2001 Inverse scattering on fractional brownian surfaces and estimation of the Hurst exponent Inverse Problems 17 365-86

[13] Adler R 1981 The Geometry of Random Fields (London: Wiley)

[14] Pesin Ya B 1993 On rigorous mathematical definition of the correlation dimension and generalized spectrum for dimension J. Stat. Phys. 71 529-47

[15] Guérin C A and Holschneider M 1997 On equivalent definitions of the correlation dimension for a probability measure J. Stat. Phys. 86 707-20

[16] Holschneider M 1994 Fractal wavelet dimension and localization Commun. Math. Phys. 160 457-73

[17] Holschneider M 1995 Wavelets, an Analysis Tool (Oxford: Oxford University Press)

[18] Bardet J M 2002 Bivariate occupation measure dimension of multidimensional processes Stoch. Process. Appl. $99323-48$ 